\title{
Regulation of storage lipid metabolism in developing and germinating lupin (Lupinus spp.) seeds
}

\author{
Sławomir Borek ${ }^{1}$ (D) $\cdot$ Wiktoria Ratajczak $^{1} \cdot$ Lech Ratajczak $^{1}$
}

Received: 18 November 2014/Revised: 15 February 2015/Accepted: 23 May 2015/Published online: 30 May 2015

(C) The Author(s) 2015. This article is published with open access at Springerlink.com

\begin{abstract}
The main storage compound in lupin seeds is protein, whose content can reach up to $45-50 \%$ of dry matter. However, seeds of some lupin species can also contain quite a large amount of storage lipid. The range of lipid content in lupin seeds is from about 6 to about $20 \%$ of dry matter. Storage lipid in developing seeds is synthesized mainly from sugars delivered by mother plants. During seed germination, one of the main end-products of storage lipid breakdown is also sugars. Thus, the sugar level in tissues is considered an important regulatory agent, during both lipid accumulation and lipid breakdown. Generally, in developing legume seeds, there is a strong negative relation between accumulation of storage protein and storage lipid. Results obtained in developing lupin cotyledons cultured in vitro pointed to the possibility of a positive relation between protein and lipid accumulation. Such a positive effect could be caused by nitrate. During lupin seed germination and seedling development, the utilization of storage lipid is enhanced under sugar deficiency conditions in tissues and is controlled at the gene expression level. However, under sugar starvation conditions, autophagy is significantly enhanced, and it can cause disturbances in storage lipid breakdown. The hypothesis of pexophagy, i.e., autophagic degradation of peroxisomes under sugar starvation conditions during lupin seed germination, has been taken into consideration. The flow of lipid-derived carbon skeletons to amino acids was
\end{abstract}

Communicated by A. K. Kononowicz.

Sławomir Borek

borek@amu.edu.pl

1 Faculty of Biology, Department of Plant Physiology, Adam Mickiewicz University, ul. Umultowska 89, 61-614 Poznań, Poland discovered in germinating lupin seeds, and this process is clearly more intense in sucrose-fed embryo axes. At least four alternative or mutually complementary pathways of carbon flow from storage lipid to amino acids in germinating lupin seeds are postulated. The different strategies of storage compound breakdown during lupin seed germination are also discussed.

Keywords Asparagine $\cdot$ Nitrate $\cdot$ Non-starch seed Protein seed · Sucrose

\section{Introduction}

The main storage compound in mature lupin seeds is protein. Yellow lupin (Lupinus luteus L.) seeds, for example, can contain about $45 \%$ protein in dry matter (Cerletti 1982). Similarly, a high level of protein occurs in Andean lupin (L. mutabilis Sweet) seeds, and its content can reach 40-50\% (Santos et al. 1997). Lupin seeds contain two classes of proteins which, according to Osborn's classification, correspond to albumin and globulin fractions (Duranti et al. 2008). The globulins dominate in lupin seeds. In yellow lupin, they might constitute up to $80 \%$ of the total seed protein content (Gwóźdź 1988; Ratajczak et al. 1999). In white lupin (L. albus L.) seeds, the albumin to globulin ratio is about 1/9 (Duranti et al. 2008), and in Andean lupin seeds this ratio is about $1 / 5$ (Santos et al. 1997). Lupin seeds can also contain large quantities of storage lipid. An example of low lipid content is yellow lupin seeds, which contain only about $6 \%$ of lipid in dry matter. Opposite to yellow lupin is Andean lupin, whose seeds contain the highest lipid amount among lupins, and the level of storage lipid can reach $20 \%$ (Borek et al. 2009). In this case, Andean lupin seeds are similar to soybean seeds, which can 
contain 12-26\% storage lipid (Zhou et al. 2006). Seeds of white lupin contain 7-14 \% lipid in dry matter (Borek et al. 2009). Carbohydrate content in lupin seeds is on average about $36 \%$ of dry matter, of which $26 \%$ is fiber (Hedley 2001). Mature, dry lupin seeds contain no starch (Borek et al. 2006; Duranti et al. 2008; Borek et al. 2011, 2013a). However, starch temporarily occurs during lupin seed development, disappears during seed maturation and desiccation, and reappears during seed imbibition and germination (Borek et al. 2013a). The comparison of seed chemical composition of several agriculturally important plants (including lupins) is presented in Table 1.

In the research on mechanisms of regulation of storage lipid accumulation and storage lipid breakdown, sucrose is primarily considered. It is the substrate for storage lipid biosynthesis in developing seeds (Weber et al. 2005; Baud et al. 2008) and is one of the main end-products of storage lipid breakdown during seed germination (Graham 2008; Quettier and Eastmond 2009). Sucrose is also an important regulatory agent that by modifications in expression of many genes can control among others the synthesis and degradation of storage compounds (Smeekens et al. 2010; Morkunas et al. 2012). Other factors taken into account in the studies on lipid metabolism in lupin seeds are asparagine and nitrate. Asparagine is an essential amino acid in the nitrogen metabolism of lupin seeds (Ratajczak 1986). For example, in developing soybean seeds, this amino acid can constitute from 33 to $49 \%$ of the free amino acid pool and enhances storage protein accumulation (HernándezSebastià et al. 2005). In lupin germinating seeds, the level of asparagine can reach $30 \%$ of dry matter, and it is a main transport form of nitrogen from source to sink tissues (Lehmann and Ratajczak 2008). In white lupin, asparagine comprises $55-70 \%$ of the nitrogen of xylem sap and $40-60 \%$ of the nitrogen of stem base phloem sap. Organs with high proportions of their total nitrogen as free amino compounds have very high proportions (up to $80 \%$ ) of their soluble amino-N as asparagine (Pate et al. 1981). And finally, this amino acid influences carbohydrate metabolism in developing and germinating lupin seeds. Asparagine significantly reduces the soluble sugar level and simultaneously increases starch content in organs of lupin seeds (Borek et al. 2013a). Nitrate, in contrast to asparagine, is not a favorable source of nitrogen in protein metabolism in lupin seeds (Ratajczak et al. 1988, 1996) and rather does not influence the carbohydrate metabolism in lupin seeds (Borek et al. 2013a). However, this inorganic kind of nitrogen, similarly as sucrose, is regarded as a factor which can regulate plant metabolism by changes in the expression of some genes (Vidal and Gutiérrez 2008). According to
Table 1 Seed chemical composition of agriculturally important plants (average values)

\begin{tabular}{|c|c|c|c|}
\hline \multirow[t]{2}{*}{ Species or genus } & \multicolumn{3}{|c|}{ Content ( $\%$ of seed dry matter) } \\
\hline & Protein & Lipid & Carbohydrates (total) \\
\hline Andean lupin & $40-50$ & 20 & 36 (including $26 \%$ of fiber no starch) \\
\hline Yellow lupin & 45 & 6 & \\
\hline White lupin & 38 & $7-14$ & \\
\hline Narrow-leaf lupin & 31 & $6-10$ & \\
\hline Soybean & $37-44$ & $12-26$ & 30-35 (mainly as a fiber) \\
\hline Pea & $24-30$ & $3-6$ & $56-60$ (including $51 \%$ of starch) \\
\hline Beans & $23-32$ & 1 & 56 \\
\hline Lentil & 30 & 3 & 62 \\
\hline Peanut & 31 & $48-50$ & $12-14$ \\
\hline Sunflower & 25 & 49 & 1 \\
\hline Flax & 24 & 36 & 24 \\
\hline Rape & 21 & 48 & 19 \\
\hline Castor bean & 18 & 64 & 1 \\
\hline Oat & $9-13$ & $6-8$ & 66 \\
\hline Maize & $9-11$ & $4-5$ & $66-75$ \\
\hline Pearl millet & 11 & $4-5$ & $65-72$ \\
\hline Barley & 11 & $2-3$ & $60-72$ \\
\hline Wheat & $10-12$ & 2 & $71-75$ \\
\hline Rye & $9-12$ & 2 & $73-75$ \\
\hline Rice & 9 & $1-2$ & 74 \\
\hline Sorghum & 8 & 4 & 67 \\
\hline Brown rice & 7 & 2 & 65 \\
\hline
\end{tabular}


current knowledge, the regulatory function of nitrate focuses on the root meristem activity (Crawford and Forde 2002; Liu et al. 2008), nitrate transport, reduction and assimilation (Vidal and Gutiérrez 2008; Alvarez et al. 2012), and expression of genes of central metabolism, for example, genes of some enzymes involved in the Krebs cycle (malate dehydrogenase and isocitrate dehydrogenase) (Aceituno et al. 2008).

\section{Regulation of storage lipid accumulation in developing lupin seeds}

Storage lipid in developing seeds is synthesized mainly from sugars (mostly from sucrose and glucose) and to a lesser extent from amino acids provided by the mother plant (Weber et al. 2005; Baud et al. 2008). Sugars in lipidstoring cells undergo glycolysis, which occurs both in cytoplasm and in plastids. Within plastids, acetyl-CoA, a direct substrate for fatty acid biosynthesis, is synthesized. In plastids, the most common fatty acids, such as palmitic acid, stearic acid and oleic acid, are mainly synthesized. Lengthening and other modifications of fatty acids already occur in the endoplasmic reticulum. In this organelle, through the Kennedy pathway, triacylglycerols (the main component of storage lipid) are synthesized from fatty acids and glycerol-3-phosphate. Finally, storage lipid is deposited in cytoplasm in the form of oil bodies (oleosomes) (Fig. 1).

Experiments conducted on developing lupin cotyledons cultured in vitro showed that sucrose significantly enhances lipid accumulation. A similar, but less evident, effect was observed regarding protein content (Borek et al. 2009). Asparagine, as a 'central' amino acid in nitrogen metabolism of lupin seeds, significantly enhanced accumulation of protein, but simultaneously reduced lipid content. However, the key result was an effect caused by nitrate, which simultaneously increased the content of both lipid and protein. The stimulatory effect of nitrate was more evident in cotyledons fed with sucrose than in those not fed with sugar. Nitrate caused an increase in lipid content by $7.8 \%$ in sucrose-fed yellow lupin cotyledons, by $8.6 \%$ in white lupin cotyledons, and by $10.2 \%$ in Andean lupin cotyledons. The simultaneous increase in protein content was 16.1, 9.3 and $11.4 \%$, respectively. To explain the mechanism of the stimulatory effect of nitrate, the data obtained from experiments with specifically ${ }^{14} \mathrm{C}$-labeled acetate $(1-$ and $2-{ }^{14} \mathrm{C}$-acetic acid) were necessary. Acetate after entering the cell is activated to acetyl-CoA (Turner et al. 2005; Hooks et al. 2007) and can be a substrate for storage lipid biosynthesis. Analysis of radioactivity of the lipid fraction of isolated cotyledons cultured in vitro showed significantly lower labeling in organs fed with nitrate. Comparing this result with the higher lipid content, one may suspect that during enhanced lipid biosynthesis from non-labeled substrate (sucrose) the radioactivity of the accumulated lipid is finally decreased. Based on such results and literature data showing that nitrate enhances glycolytic efficiency in embryos of Brassica napus (Junker et al. 2007), the hypothesis was formulated that the stimulation by nitrate of storage lipid synthesis in developing lupin seeds is due to the intensification of the flow of carbon through the process of glycolysis. In such circumstances, a larger amount of acetyl-CoA is synthesized, and in turn it leads to enhanced lipid accumulation (Borek et al. 2009).

The above-described results are the only ones in the scientific literature which point to the possibility of a simultaneous increase in lipid and protein content in maturing legume seeds. There are many papers describing a completely opposite relationship in legume seeds, i.e., a strong negative relationship in lipid and protein accumulation. This negative relationship was discovered already in the middle of the last century, and has been confirmed many times since, especially in soybean seeds (Chung et al. 2003). The same negative relationship in developing lupin cotyledons was caused by asparagine (Borek et al. 2009). The discovery that it is possible to achieve a positive relationship in lipid and protein accumulation by the simple manipulation of trophic conditions of in vitro culture is especially important because it brings understanding of an important part of the regulatory mechanisms operating in maturing seeds. It also encouraged further research to verify whether the in vitro observed effect of nitrate would be reflected in field conditions, because achieving this goal would have a high economic importance. Unfortunately, experiments executed later on developing yellow lupin pods grown in quasi in vitro culture (the culture was conducted under not fully sterile conditions), and experiments conducted under field conditions, showed that the positive effect of nitrate is only available in isolated developing lupin cotyledons which are cultured under artificial in vitro conditions (Borek et al. 2014). Experiments conducted on the developing pods showed that the positive relationship caused by nitrate (as well as the negative relationship caused by asparagine) was the same as that observed in isolated cotyledons cultured in vitro, but the effect caused by the trophic conditions of in vitro culture was remarkably less evident. And no effect of nitrate was observed under field conditions. Application of nitrate fertilizer (40 or $80 \mathrm{~kg}$ of $\mathrm{N}$ per ha) did not cause changes in lipid and protein content in mature lupin seeds (Borek et al. 2014). The measurement of nitrate level in particular parts of developing lupin pods (wall of pod, seed cover and embryo) showed that this inorganic kind of nitrogen is accumulated in the wall of the pod, and its level in developing cotyledons inside the pod remains low and is only slightly 
Fig. 1 Schematic

representation of storage lipid biosynthesis in plants, based on Baud et al. (2008) and Li-

Beisson et al. (2010). The main substrates for lipid biosynthesis are sugars which are supplied by the mother plant. Amino acids are also necessary. Glutamine is the main transport form of nitrogen in majority of plants. However, asparagine is the most important amino acid in transportation of nitrogen in legumes. Sugars undergo cytosolic and plastidic (chloroplastic) glycolysis inside the cells of storage organs.

Arising malonyl-CoA delivers two-carbon units for fatty acids biosynthesis. Mostly the typical and widespread fatty acids are synthesized in plastids.

Processes such as fatty acid elongation, desaturation and other modifications take place in endoplasmic reticulum. In the same compartment, triacylglycerols are synthesized from fatty acids and glycerol-3phosphate. Finally,

triacylglycerols as the main storage lipid compound are deposited inside oil bodies, which are scattered in the cytosol. ACP acyl-carrier protein, $D A G$ diacylglycerol, DHAP dihydroxyacetone phosphate, G-6-P glucose-6phosphate, $P A$ pyruvate, $P E P$ phosphoenolpyruvate, $T A G$ triacylglycerol

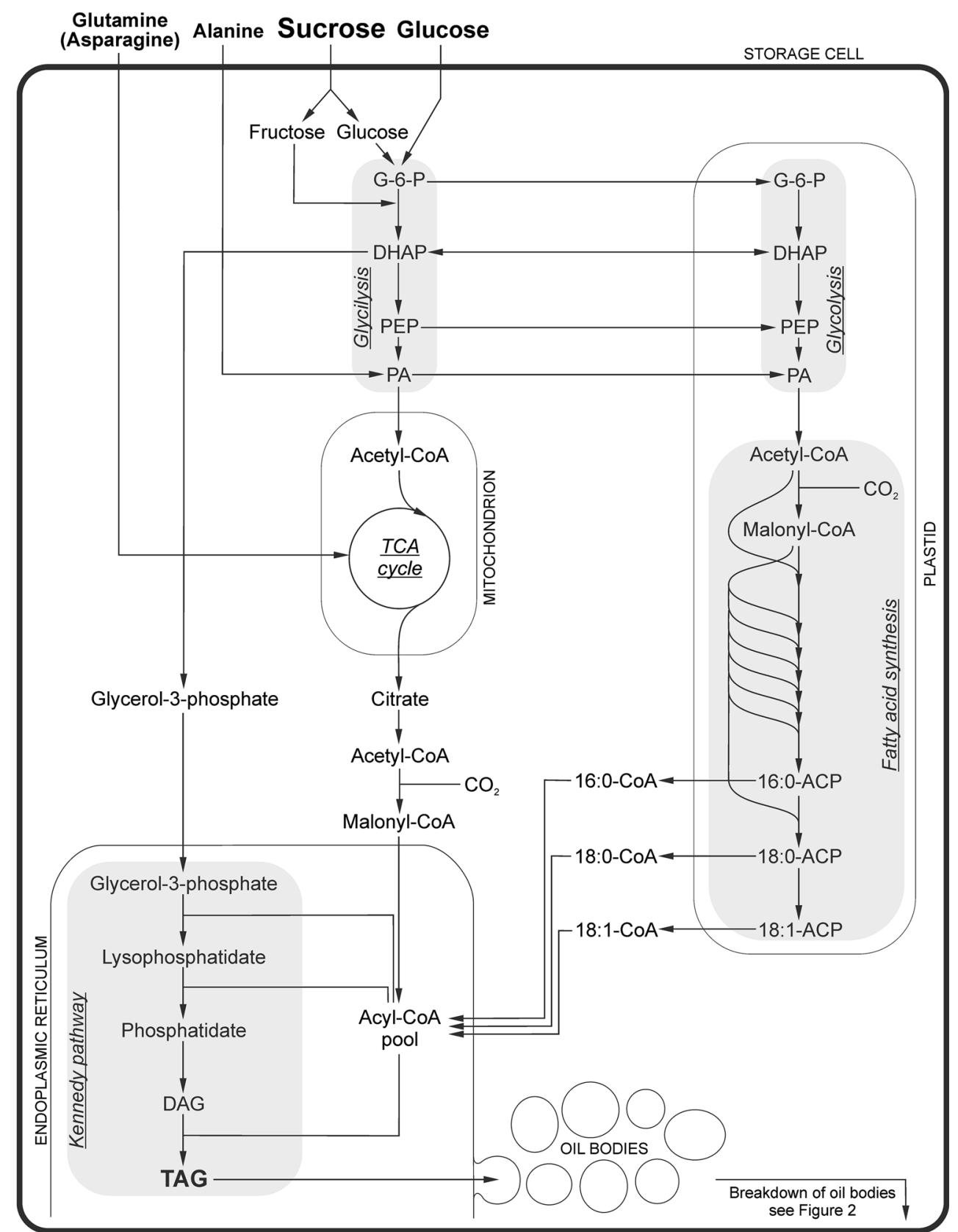

elevated by exogenously applied nitrate. Only application of nitrate to isolated cotyledons cultured in vitro causes the positive relationship in storage lipid and protein accumulation, because nitrate can be taken up by cotyledons and can enter the cells where storage compounds are synthesized (data not published). Probably in pods there exists a mechanism involving specific transporters which precisely control the content of the nutritional cocktail loaded from the mother plant to the developing seeds.

During the research conducted on developing lupin seeds (Borek et al. 2009), special attention was also paid to the properties of lupin oil. Yellow lupin oil contains a high amount of linoleic acid, similarly as soybean and sunflower oils. White lupin oil contains a high amount of oleic acid and in this respect is similar to canola oil. However, the most interesting feature is that Andean lupin oil contains high amounts of oleic and linoleic acids, combining features of soybean, canola, and sunflower oils. Moreover, Andean lupin oil does not contain erucic acid, which is harmful for the human organism. Lupin oils also contain a high amount of phosphatidylcholine, i.e., lecithin. These properties of lupin oils increase the potential for industrial use of lupin seeds to produce oil with high quality parameters that are relevant in the human diet. 


\section{Regulation of storage lipid breakdown in germinating lupin seeds}

The mobilization of storage lipid during seed germination begins with the decomposition of the triacylglycerols accumulated in oil bodies into free fatty acids and glycerol. Mostly lipases are active at this stage of the process (Barros et al. 2010). Next, the fatty acids undergo $\beta$-oxidation, which occurs in peroxisomes (glyoxysomes). The following step is the glyoxylate cycle, which partially occurs in the peroxisome and partially in the cytoplasm. In the peroxisome are located three of the five enzymes of the glyoxylate cycle (citrate synthase, isocitrate lyase and malate synthase), while two other enzymes (aconitase and malate dehydrogenase) operate in the cytoplasm (Pracharoenwattana and Smith 2008). Succinate synthesized in the peroxisome, after transport into the mitochondrium, is converted to malate through a part of the Krebs cycle. This metabolite, in turn, after transport to the cytoplasm, is converted to oxaloacetate. The last step of storage lipid breakdown is gluconeogenesis and the synthesis of sugars, which are a form of carbon transport in plants (Quettier and Eastmond 2009; Borek and Ratajczak 2010) (Fig. 2).

Since sugars are one of the end-products of storage lipid breakdown during seed germination, the regulatory function of sucrose is primarily investigated. The in vitro culture of lupin embryo axes and cotyledons isolated from imbibed seeds and the in vitro culture of whole lupin seedlings are very useful in the research. By the addition of sucrose to the culture medium or by a lack of sugar in the medium, it is possible to manipulate the sugar level in tissues of organs of germinating lupin seeds (Borek et al. 2006). Ultrastructural observations of cells (Borek et al. 2006, 2011, 2012a) and the measurement of lipid content (Borek et al. 2012b) in lupin seedlings and isolated embryo axes and cotyledons cultured in vitro for $96 \mathrm{~h}$ showed that the sugar deficit in tissues enhances lipid breakdown only in seedlings and in isolated cotyledons. A completely opposite relationship was observed in lupin sugar-starved isolated embryo axes cultured for $96 \mathrm{~h}$ in vitro. In these organs, the lipid content was significantly higher than in axes fed with sucrose (Borek et al. 2011, 2012b). The total lipid content in sugar-starved isolated embryo axes of yellow lupin cultured in vitro was higher by $43 \%$ than in the sucrose-fed axes; in axes of white lupin, it was higher by $44 \%$, and in axes of Andean lupin it was higher by $70 \%$ (Borek et al. 2012b). This result is completely inconsistent with numerous other literature data, and the mechanism of such an effect remains unexplained. In the literature, there is described a considerable intensification of breakdown of storage compounds in sugar starvation conditions (exactly as was observed in cultured in vitro lupin seedlings and isolated cotyledons) (Morkunas et al. 2012). It was noted, however, that in sugar starvation conditions autophagy occurs in isolated lupin embryo axes. Symptoms of autophagy were a huge increase in cell vacuolization (Borek et al. 2006, 2011) and a significant decrease in phosphatidylcholine content (Borek et al. 2012b). Based on such results, the following hypothesis was formulated. Autophagy causes disturbances in action of the enzymes involved in storage lipid breakdown. More precisely, in conditions of sugar starvation, the peroxisomes could be degraded during autophagy. This causes that the breakdown of storage lipid in sugar-starved embryo axes can be disrupted and more lipid remains in sugarstarved axes compared to the sugar-fed organs. In cells of axes fed with sucrose, autophagy does not occur; they grow intensely and lipid reserves are utilized in larger quantities (Borek et al. 2011, 2012b). The above-presented hypothesis was recently supported by ultrastructural observations of cells of sugar-starved isolated embryo axes which were simultaneously fed with asparagine. This amino acid remarkably slows down the decomposition of autophagic bodies in sugar-starved lupin embryo axes, and this allows identification of the cell organelles which are degraded during autophagy. Among them were peroxisomes. Such results support another hypothesis that in sugar-starved lupin embryo axes, pexophagy (a special kind of autophagy during which the peroxisomes are selectively degraded) can occur (Borek et al. 2013c).

Note, however, that the above-described special regulatory role of sucrose in lupin-isolated embryo axes is manifested only at the level of cell ultrastructure and at the level of analytical determinations of lipid content in the examined organs. Nevertheless, the changes observed at the level of enzymes activity, mRNA content, phospholipid content, and fatty acid spectra tend to fit into a more general picture of changes in lipid metabolism caused by changing sugar content in tissues-a picture that is consistent for both isolated embryo axes and for isolated cotyledons and organs of seedlings. Moreover, this regulatory role of sugars in the mobilization of storage lipid is based on changes in the expression of genes encoding enzymes involved in pathways of storage lipid breakdown (Borek and Nuc 2011).

Analyzing changes in enzyme activities and mRNA levels for selected enzyme proteins, it can be stated that in sugar deficiency or starvation conditions in tissues there occurs intensification of storage lipid breakdown, but only the initial stages of this process are enhanced (Fig. 3). Examples include a marked increase in sugar starvation conditions of activity and mRNA level of lipase (lipolysis) and the increase in activity of acyl-CoA oxidase and catalase ( $\beta$-oxidation of fatty acids) (Borek and Nuc 2011; 
Fig. 2 Schematic representation of storage lipid breakdown in plants, based on Borek and Ratajczak (2010), Borek et al. (2013b) and literature cited there. During seed germination, as well as during seedling growth, triacylglycerols (TAG) are decomposed mainly by lipases. Liberated fatty acids undergo $\beta$ oxidation in the peroxisome (called also a glyoxysome in oilseeds). This process generates acetyl-CoA which during the glyoxylate cycle (that occurs both in the peroxisome and in the cytosol) is converted to succinate. Next, through part of the tricarboxylic acid (TCA) cycle succinate is converted to malate. The last step of storage lipid mobilization is synthesis of sugars through the

gluconeogenesis pathway.

Sucrose as the main carbon transport form is exported to other, non-storing tissues of developing seedling

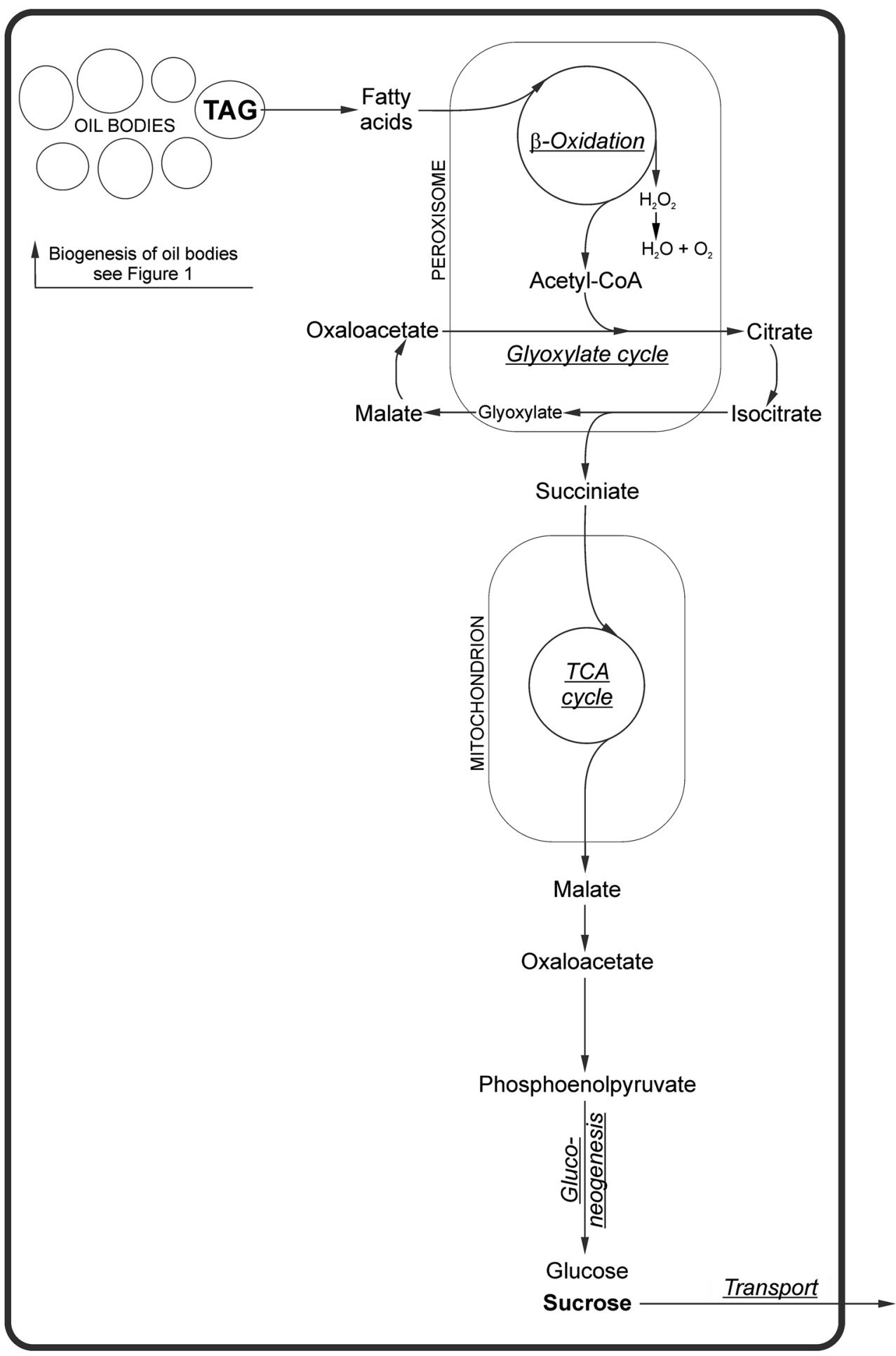

Borek et al. 2013b). In turn, the activity and mRNA level of cytosolic aconitase, isocitrate lyase activity (glyoxylate cycle) and the activity of phosphoenolpyruvate carboxykinase (gluconeogenesis) were significantly higher in organs (especially in isolated lupin embryo axes) fed with sucrose (Borek and Nuc 2011; Borek et al. 2013b). Also, activity and mRNA level of cytosolic isocitrate dehydrogenase were higher in sucrose-fed embryo axes (Borek et al. 2006; Borek and Nuc 2011) (Fig. 3). These results are completely opposite to the numerous literature data which show increased activity and gene expression of enzymes involved in breakdown of storage compounds, or generally involved in catabolic processes in conditions of low sugar level in tissues. However, an explanation of 


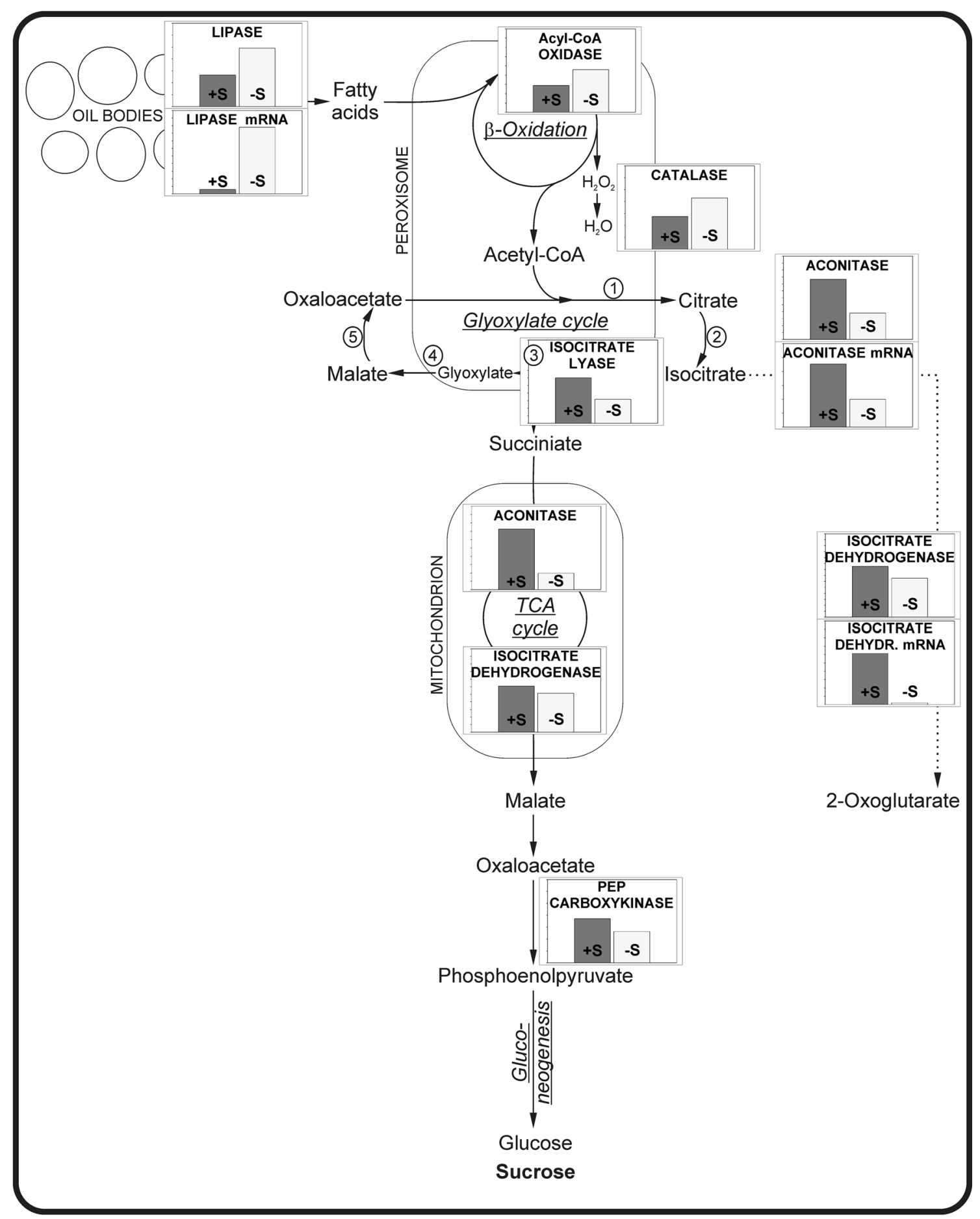

Fig. 3 Activity and mRNA level for selected enzymes involved in storage lipid breakdown in yellow lupin embryo axes which were isolated from imbibed seeds and next were grown in vitro for $96 \mathrm{~h}$ in darkness on liquid mineral medium supplemented with sucrose $(+S)$ or without the sugar $(-S)$, based on Borek and Nuc (2011) and Borek et al. $(2006,2013 b)$. Breakdown of storage lipid is initiated by lipases which are active on the surface of oil bodies. Acyl-CoA oxidase is the enzyme which introduces fatty acids into the $\beta$-oxidation cycle. During this cycle $\mathrm{H}_{2} \mathrm{O}_{2}$ is also generated, and this toxic kind of oxygen is decomposed by catalase. The glyoxylate cycle is catalyzed by five enzymes, i.e., by citrate synthase $(1$; operates inside the peroxisome), aconitase (2; operates in the cytosol), isocitrate lyase (3; peroxisome), malate synthase (4; peroxisome), and malate dehydrogenase (5; cytosol). Aconitase is also one of the enzymes of the TCA cycle. Phosphoenolpyruvate (PEP) carboxykinase starts the gluconeogenesis pathway. In tissues of germinating lupin seeds, isocitrate dehydrogenase is active. This is an important enzyme in one of the newly described pathways of carbon flow from fatty acids to amino acids in lupine tissues (Fig. 4). By the action of this enzyme, the lipidderived carbon atoms could be subtracted from the typical pathway of storage lipid breakdown and could be directed to amino acids synthesis 
these lupin-specific results was possible upon obtaining data from experiments in vivo with specifically ${ }^{14} \mathrm{C}$-labeled acetate (1- and $2-{ }^{14} \mathrm{C}$-acetic acid). In experiments on storage lipid breakdown during seed germination, exogenously applied acetate is used as a precursor of fatty acid. Part of it can be directed into the peroxisome and is activated by acetyl-CoA synthetase (AcetCS) (Turner et al. 2005; Hooks et al. 2007). In such a way, acetate can be included in pathways of storage lipid breakdown. These studies led to the discovery of strong links between pathways of storage lipid and pathways of storage protein breakdown (Borek et al. 2003; Borek and Ratajczak 2010). It turns out that during lupin seed germination the flow of lipid-derived carbon skeletons to amino acids (mainly asparagine, glutamine and glutamate) occurs, and this flow is clearly more intense in sucrose-fed axes. Isolated lupin embryo axes (as well as isolated cotyledons) fed with sucrose grow more intensely than organs not fed with sucrose, and the lipidderived carbon skeletons can complement the source of organic carbon in conditions of enhanced biosynthesis of amino acids. The results allowed four alternative or mutually complementary pathways of carbon flow from storage lipid to amino acids in germinating lupin seeds to be described (Borek and Ratajczak 2010) (Fig. 4). Probably, the carbon skeletons 'leave' the classic (described for germinating oilseeds) pathway of storage lipid breakdown at the stage of the glyoxylate cycle. As mentioned above, two of five enzymes of this cycle operate in the cytoplasm. They are aconitase and malate dehydrogenase. Therefore, the substrate and product of aconitase (citrate and isocitrate, respectively) and the substrate and product of malate dehydrogenase (malate and oxaloacetate, respectively) must appear in the cytoplasm (Pracharoenwattana and Smith 2008). Thus, these compounds can 'leave' the glyoxylate cycle, and more or less directly can be used for the synthesis of amino acids, which occurs in the cytoplasm. In addition to the cytosolic enzymes of the glyoxylate cycle, also of importance in these pathways is the cytosolic isocitrate dehydrogenase, which catalyzes the conversion of isocitrate to 2-oxoglutarate (Borek et al. 2006, 2011). As already mentioned, the carbon flow from fatty acids to amino acids was more intense in the sucrosefed axes, and the activity and mRNA level of this enzyme were also elevated in conditions of good supply of tissue with sugars (Borek et al. 2006; Borek and Nuc 2011) (Fig. 3). The existence of pathways of asparagine biosynthesis is especially important for legumes because in these plants the nitrogen metabolism is intensive. In germinating lupin seeds, amino acids liberated from storage proteins are used as respiratory substrates (Borek et al. 2001; Lehmann and Ratajczak 2008), and during deamination reactions toxic ammonia is generated. To protect tissues from ammonia poisoning, asparagine is synthesized (Lehmann and
Ratajczak 2008). The above-mentioned pathways of carbon flow from fatty acids to asparagine in germinating lupin seeds are additionally important in preventing ammonia poisoning because they supply the precursors (oxaloacetate and aspartic acid) for asparagine biosynthesis.

The majority of the research described in this review was simultaneously conducted on seeds of three lupin species (Borek et al. 2009, 2011, 2012a, b, 2013a, b) which significantly differ regarding storage lipid content. They were seeds of yellow lupin (lipid content about $6 \%$ in seed dry matter), white lupin (lipid content 7-14\%) and Andean lupin (lipid content about $20 \%$ ). The results obtained have shown that the regulation of lipid metabolism in lupin seeds seems to be independent of the amount of this storage compound in seeds. This concerns both the regulation of lipid biosynthesis in developing seeds and lipid degradation during lupin seed germination. The regulatory role of sucrose (as well as asparagine and nitrate) is almost identical in all three investigated lupin species. However, the lipid content, or rather the relative proportions of storage lipid and protein contents, is important in a broader sense of regulation of storage compound mobilization during lupin seed germination. Discussing this process in germinating lupin seeds, it should be kept in mind that protein is the main storage compound in these species. Protein content in mature, dry yellow lupin seeds can reach about $45 \%$ of dry matter (Cerletti 1982); in white lupin seeds, its content can reach $38 \%$ (Mohamed and Rayas-Duarte 1995), and in Andean lupin seeds protein content can vary from 40 to $50 \%$ (Santos et al. 1997). Mature, dry lupin seeds do not contain starch (Borek et al. 2011, 2013a). Research using specifically ${ }^{14} \mathrm{C}$-labeled acetate and a glutamine synthetase inhibitor (MSO, L-methionine sulfoximine) pointed to different strategies in mobilization of storage compounds during germination of seeds of investigated lupin species (Borek et al. 2011). In germinating yellow lupin seeds, characterized by a much higher content of storage protein than storage lipid, the main metabolites are amino acids. Amino acids are used in many anabolic processes, but they are also important respiratory substrates in germinating lupin seeds (Borek et al. 2001; Lehmann and Ratajczak 2008). Disturbances in amino acid metabolism in organs of germinating yellow lupin seeds (caused by MSO) do not cause any significant changes in the intensity of breakdown of small quantities of storage lipid. In the case of germinating white lupin seeds, starch appears in axes and cotyledons already at the stage of seed imbibition (Borek et al. 2011, 2013a). Thus, there is an additional source of substrates used in anabolic processes and in respiration. In such circumstances, the disturbances in amino acid metabolism (by MSO) also did not result in clear intensification of storage lipid breakdown. However, a completely different strategy was observed in 


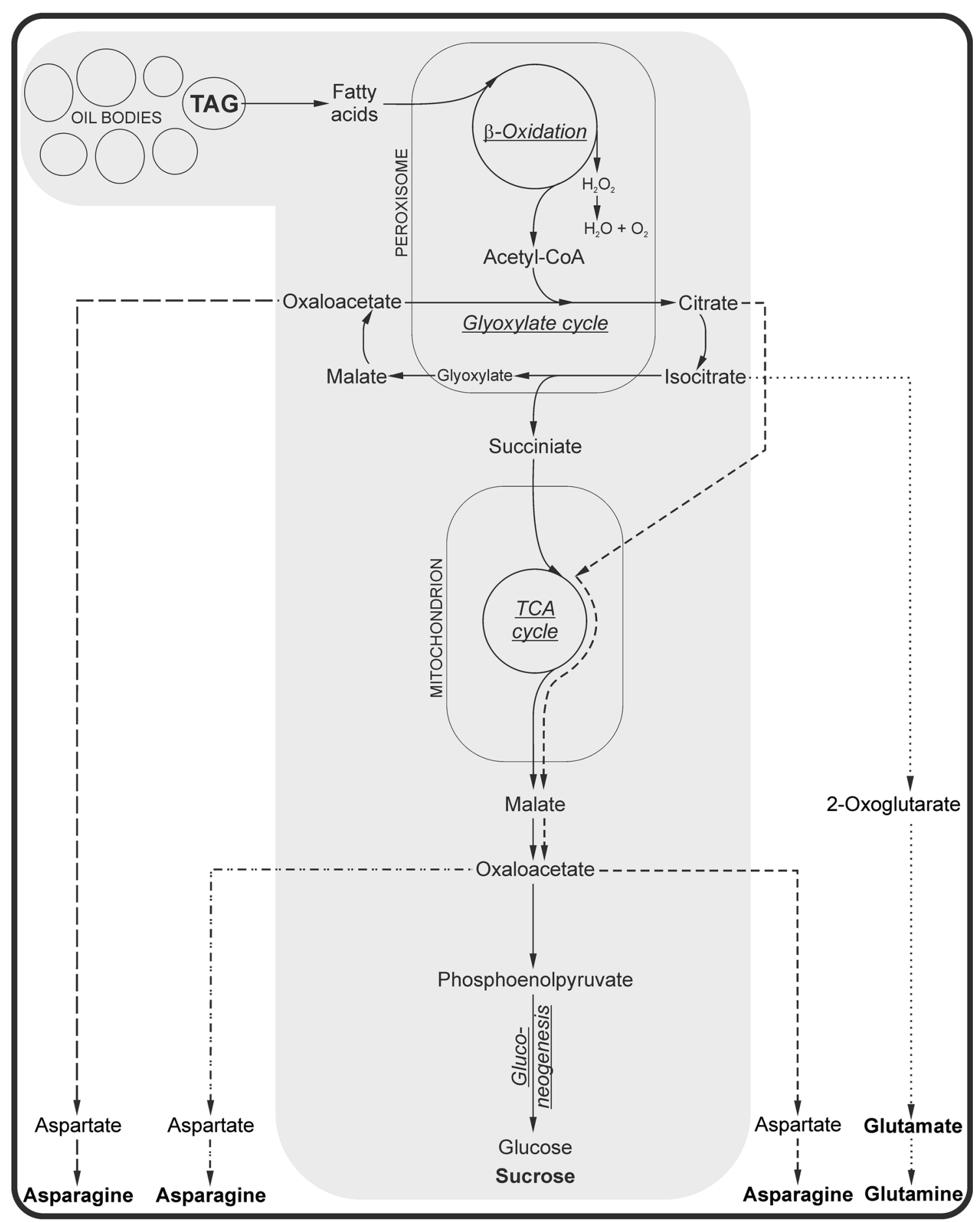

Fig. 4 Schematic representation of alternative pathways of carbon flow from fatty acids to amino acids in germinating seeds of yellow lupin, based on Borek et al. (2003) and Borek and Ratajczak (2010). The typical pathway of storage lipid conversion into sugars in plants (existing also in lupin tissues) is marked by the gray background and solid-line arrows. However, in tissues of germinating lupin seeds, the

germinating Andean lupin seeds. Seeds of this species contain large quantities of both protein and lipid. The high lipid content in the seeds must be connected with the more or less direct carbon flow from storage lipid to amino acids was discovered. At least four alternative or mutually complementary pathways exist in lupin tissues (marked by dashed-line and dotted-line arrows). Cytosolic intermediates of the glyoxylate cycle are very important in the newly described pathways. TAG, triacylglycerol

proper functioning of the enzymatic apparatus which allows the breakdown of this important storage compound. Therefore, disturbances in amino acid metabolism (due to 
application of MSO) and thus decreasing intensity of storage protein breakdown were quickly and very clearly compensated by more intense decomposition of storage lipid. In such circumstances, fatty acids can become important substrates in anabolic processes and respiratory substrates. Such a strategy is possible even in the deficit of sugar in tissues (Borek et al. 2011). The discovery of different strategies of storage compound mobilization during lupin seed germination is an important result of the abovedescribed comparative experiments.

Author contribution statement All co-authors have equal contribution to this article.

Acknowledgments This work was supported by the Polish National Science Centre (NCN; Grant Number N N310 003540).

Open Access This article is distributed under the terms of the Creative Commons Attribution 4.0 International License (http:// creativecommons.org/licenses/by/4.0/), which permits unrestricted use, distribution, and reproduction in any medium, provided you give appropriate credit to the original author(s) and the source, provide a link to the Creative Commons license, and indicate if changes were made.

\section{References}

Aceituno FF, Moseyko N, Rhee SY, Gutiérrez RA (2008) The rules of gene expression in plants: organ identity and gene body methylation are key factors for regulation of gene expression in Arabidopsis thaliana. BMC Genom 9:438. doi:10.1186/14712164-9-438

Alvarez JM, Vidal EA, Gutiérrez RA (2012) Integration of local and systemic signaling pathways for plant $\mathrm{N}$ responses. Curr Opin Plant Biol 15:185-191

Barros M, Fleuri LF, Macedo GA (2010) Seed lipases: sources, applications and properties-a review. Braz J Chem Engin 27:15-29

Baud S, Dubreucq B, Miquel M, Rochat C, Lepiniec L (2008) Storage reserve accumulation in Arabidopsis: metabolic and developmental control of seed filling. Arabidopsis Book Am Soc Plant Biolog. doi:10.1199/tab.0113

Borek S, Nuc K (2011) Sucrose controls storage lipid breakdown on gene expression level in germinating yellow lupine (Lupinus luteus L.) seeds. J Plant Physiol 168:1795-1803

Borek S, Ratajczak L (2010) Storage lipids as a source of carbon skeletons for asparagine synthesis in germinating seeds of yellow lupine (Lupinus luteus L.). J Plant Physiol 167:717-724

Borek S, Morkunas I, Ratajczak W, Ratajczak L (2001) Metabolism of amino acids in germinating yellow lupin seeds. III. Breakdown of arginine in sugar-starved organs cultivated in vitro. Acta Physiol Plant 23:141-148

Borek S, Ratajczak W, Ratajczak L (2003) A transfer of carbon atoms from fatty acids to sugars and amino acids in yellow lupine (Lupinus luteus L.) seedlings. J Plant Physiol 160:539-545

Borek S, Ratajczak W, Ratajczak L (2006) Ultrastructural and enzymatic research on the role of sucrose in mobilization of storage lipids in germinating yellow lupine seeds. Plant Sci 170:441-452

Borek S, Pukacka S, Michalski K, Ratajczak L (2009) Lipid and protein accumulation in developing seeds of three lupine species:
Lupinus luteus L., Lupinus albus L., and Lupinus mutabilis Sweet. J Exp Bot 60:3453-3466

Borek S, Kubala S, Kubala S, Ratajczak L (2011) Comparative study of storage compounds breakdown in germinating protein-storing seeds of three lupine species of different oil content. Acta Physiol Plant 33:1953-1968

Borek S, Kubala S, Kubala S (2012a) Regulation by sucrose of storage compounds breakdown in germinating seeds of yellow lupine (Lupinus luteus L.), white lupine (Lupinus albus L.) and Andean lupine (Lupinus mutabilis Sweet). I. Mobilization of storage protein. Acta Physiol Plant 34:701-711

Borek S, Pukacka S, Michalski K (2012b) Regulation by sucrose of storage compounds breakdown in germinating seeds of yellow lupine (Lupinus luteus L.), white lupine (Lupinus albus L.) and Andean lupine (Lupinus mutabilis Sweet). II. Mobilization of storage lipid. Acta Physiol Plant 34:1199-1206

Borek S, Galor A, Paluch E (2013a) Asparagine enhances starch accumulation in developing and germinating lupin seeds. J Plant Growth Regul 32:471-482

Borek S, Kubala S, Kubala S (2013b) Diverse regulation by sucrose of enzymes involved in storage lipid breakdown in germinating lupin seeds. Acta Physiol Plant 35:2147-2156

Borek S, Paluch E, Pukacka S (2013c) Asparagine slows down the decomposition of autophagic bodies in sugar starved embryo axes of lupin (Lupinus spp). In: Figlerowicz M, Stróżycki PM (eds) 6th conference of the Polish Society of experimental plant biology. BioTechnologia JBCBB 94(3):319

Borek S, Pukacka S, Stawiński S, Nuc K (2014) Regulation of oil and protein accumulation in developing lupin seeds. In: Abstract book, poster presentations. Plant biology Europe FESPB/EPSO 2014 Congress, June 2014, Dublin, Ireland, p 390

Cerletti P (1982) Lupine seed proteins. In: Hudson BJF (ed) Developments in food proteins-2. Applied Science Publisher Ltd, London, pp 133-171

Chung J, Babka HL, Graef GL, Staswick PE, Lee DJ, Cregan PB, Shoemaker RC, Specht JE (2003) The seed protein, oil, and yield QTL on soybean linkage group I. Crop Sci 43:1053-1067

Crawford NM, Forde BG (2002) Molecular and developmental biology of inorganic nitrogen nutrition. Arabidopsis Book Am Soc Plant Biolog. doi:10.1199/tab.0011

Duranti M, Consonni A, Magni C, Sessa F, Scarafoni A (2008) The major proteins of lupin seed: characterisation and molecular properties for use as functional and nutraceutical ingredients. Trends Food Sci Technol 19:624-633

Graham IA (2008) Seed storage oil mobilization. Ann Rev Plant Biol 59:115-142

Gwóźdź EA (1988) Biosynthesis of storage proteins in developing lupin seeds. In: Twardowski T (ed) Proceedings of 5th international lupin conference. Poznań, pp 342-357

Hedley CL (2001) Grain legume carbohydrates. In: Hedley CL (ed) Carbohydrates in grain legume seeds: improving nutritional quality and agronomic characteristics. $\mathrm{CAB}$ International, Wallingford, pp 11-14

Hernández-Sebastià C, Marsolais F, Saravitz C, Israel D, Dewey RE, Huber SC (2005) Free amino acid profiles suggest a possible role for asparagine in the control of storage-product accumulation in developing seeds of low- and high-protein soybean lines. J Exp Bot 56:1951-1963

Hooks MA, Turner JE, Murphy EC, Johnston KA, Burr S, Jarosławski S (2007) The Arabidopsis ALDP protein homologue COMATOSE is instrumental in peroxisomal acetate metabolism. Biochem J 406:399-406

Junker BH, Lonien J, Heady LE, Rogers A, Schwender J (2007) Parallel determination of enzyme activities and in vivo fluxes in Brassica napus embryos grown on organic or inorganic nitrogen source. Phytochemistry 68:2232-2242 
Lehmann T, Ratajczak L (2008) The pivotal role of glutamate dehydrogenase $(\mathrm{GDH})$ in the mobilization of $\mathrm{N}$ and $\mathrm{C}$ from storage material to asparagine in germinating seeds of yellow lupine. J Plant Physiol 165:149-158

Li-Beisson Y, Shorrosh B, Beisson F, Andersson MX, Arondel V, Bates PD, Baud S, Bird D, Debono A, Durrett TP, Franke RB, Graham IA, Katayama K, Kelly AA, Larson T, Markham JE, Miquel M, Molina I, Nishida I, Rowland O, Samuels L, Schmid KM, Wada H, Welti R, Xu C, Zallot R, Ohlrogge J (2010) Acyllipid metabolism. Arabidopsis Book Am Soc Plant Biolog. doi: $10.1199 /$ tab.0133

Liu J, Han L, Chen F, Bao J, Zhang F, Mi G (2008) Microarray analysis reveals early responsive genes possibly involved in localized nitrate stimulation of lateral root development in maize (Zea mays L.). Plant Sci 175:272-282

Mohamed AA, Rayas-Duarte P (1995) Composition of Lupinus albus. Cereal Chem 72:643-647

Morkunas I, Borek S, Formela M, Ratajczak L (2012) Plant responses to sugar starvation. In: Chang C-F (ed) Carbohydratescomprehensive studies on glycobiology and glycotechnology. InTech, pp 409-438

Pate JS, Atkins CA, Herridge DF, Layzell DB (1981) Synthesis, storage, and utilization of amino compounds in white lupin (Lupinus albus L.). Plant Physiol 67:37-42

Pracharoenwattana I, Smith SM (2008) When is a peroxisome not a peroxisome? Trends Plant Sci 13:522-525

Quettier AL, Eastmond PJ (2009) Storage oil hydrolysis during early seedling growth. Plant Physiol Biochem 47:485-490

Ratajczak W (1986) Asparagine metabolism in developing seeds of Lupinus luteus L. Biochem Physiol Pflanzen 181:17-22
Ratajczak W, Gwóźdź EA, Ratajczak L (1988) Effects of asparagine and methionine on storage protein synthesis in cultured lupin cotyledons. Acta Physiol Plant 10:143-149

Ratajczak W, Gwóźdź EA, Miądowicz M (1996) Effects of nitrogen nutrition on storage protein composition yellow lupin cotyledons cultured in vitro. Acta Physiol Plant 18:295-304

Ratajczak W, Borek S, Podgórski A, Ratajczak L (1999) Variability of globulin composition in cultivars and individually tested seeds of yellow lupin (Lupinus luteus L.). Acta Physiol Plant 21:413-417

Santos CN, Ferreira RB, Teixeira AR (1997) Seeds proteins of Lupinus mutabilis. J Agric Food Chem 45:3821-3825

Smeekens S, Jingkun M, Johannes H, Rolland F (2010) Sugar signals and molecular networks controlling plant growth. Curr Opin Plant Biol 13:274-279

Turner JE, Greville K, Murphy EC, Hooks MA (2005) Characterization of Arabidopsis fluoroacetate-resistant mutants reveals the principal mechanism of acetate activation for entry into the glyoxylate cycle. J Biol Chem 280:2780-2787

Vidal EA, Gutiérrez RA (2008) A systems view of nitrogen nutrient and metabolite responses in Arabidopsis. Curr Opin Plant Biol 11:521-529

Weber H, Borisjuk L, Wobus U (2005) Molecular physiology of legume seed development. Ann Rev Plant Biol 56:253-279

Zhou S, Zhang D, Luan H, Yu F, Xin X, Hu G (2006) Primary study on protein and lipid accumulation in high oil content soybean varieties. Chin J Oil Crop Sci 28:214-216 